\title{
Biosimilar FSH Preparations Versus Highly Purified Urinary FSH for Simple Ovulation Induction in Infertile Women with Polycystic Ovary Syndrome
}

\author{
Abd El Naser Abd El Gaber Ali ${ }^{1}$, Mustafa M Khodry ${ }^{1}$, Ahmed AM Mohammad ${ }^{1}$, Mahmoud I El Rasheedy ${ }^{2}$ and \\ Ahmed M Abbas ${ }^{3 *}$ \\ ${ }^{1}$ Department of Obstetrics and Gynecology, South Valley University, Egypt
}

${ }^{2}$ Department of Obstetrics and Gynecology, Al Azhar University, Egypt

${ }^{3}$ Department of Obstetrics and Gynecology, Assiut University, Egypt

*Corresponding author: Ahmed M Abbas, Assiut University, Egypt.

Received Date: November 01, 2018

Published Date: November 13, 2018

\begin{abstract}
Objective: To compare the efficacy of biosimilar FSH preparations and highly purified urinary FSH in ovulation induction in PCOs infertile women.

Patients and Methods: 100 infertile women with PCOs had been included in the study. Patients were randomly classified according to ovulation induction drugs used into 2 groups (Group I: included 50 infertile women received biosimilar FSH preparations and Group II: included 50 infertile women received highly purified urinary FSH. All cases were monitored for follicular development by transvaginal ultrasound.

Results: There was a mildly statistically significant difference between biosimilar FSH preparations group and highly purified urinary FSH in the mean \pm SD of gonadotrophins doses used, the duration of stimulation, the number of mature follicles ( $<<0.05$ ), but there was a highly statistically significant in endometrial thickness at time of HCG triggering $(\mathrm{p}<0.01)$. There was a mildly statistically significant difference in pregnancy rates $(\mathrm{p}<0.05)$. There was a mildly statistically significant difference in development of hyperstimulation syndrome and twins' pregnancies $(\mathrm{p}<0.05)$.

Conclusion and Recommendation: Biosimilar FSH preparations were better than the highly purified urinary FSH in ovulation induction in PCOs infertile women (short duration and low doses of stimulation, significant increase in growing follicles number, enhancement in endometrial thickness and increased pregnancy rate). So, this study recommends the use of biosimilar preparations of FSH in ovulation induction in PCOs infertile women.
\end{abstract}

Keywords: Biosimilar FSH; Highly purified urinary FSH (HP-FSH); Polycystic ovary syndrome (PCOs)

\section{Introduction}

Polycystic ovary syndrome (PCOS) is considered as an endocrine disorder which affects about $5 \%$ to $10 \%$ of all females. All races and nationalities are affected and represent the commonest hormonal disorder in the women reproductive age and are considered the most common cause of female infertility [1]. For $>30$ years, gonadotropins have been used a crucial element in pharmacological ovarian stimulation drugs in assisted reproductive technology (ART) in the primary and secondary infertility and in the treatment of hypogonadotropic hypogonadism [2].
Highly purified (HP) urinary FSH has been manufactured by the use of the monoclonal antibodies which is specific to FSH. Highly purified urinary FSH preparations contain < $0.1 \mathrm{IU}$ LH and the FSH specific activity is $10,000 \mathrm{IU} / \mathrm{mg}$ protein, it contains less than $5 \%$ unidentified urinary proteins [3]. Lack of urinary proteins in HPuFSH diminishes the adverse reactions like hypersensitivity or local Allergy [4,5], while LH absence has no negative impact in the ovarian stimulation in PCOS infertile women $[6,7]$. 
Biosimilars were described by the FDA as biologic products which are highly similar to reference product not withstanding few differences in the clinically inactive components, so there are no clinically clear differences between the biologic products and the reference products as regard to the safety, the purity, and the potency of these products [8]. This above-mentioned definition clearly detects that biosimilars are not identical generics or molecules for the biologic agents. They still differ in the strength, the purity and also contain different composition of the isoforms and/ or the various glycosylation profiles, with subsequent alterations in the clinical efficacy or safety of these agents [9].

The chemical structure of a generic small molecule drug should be identical to the chemical structure of its reference product, so when chemical identity had been demonstrated, approval of the generic drug may be relying upon a study that demonstrate the pharmacokinetic equivalence to reference drug, so subsequent comparative clinical trials for efficacy demonstration of the reference drug equivalent are not required [10]. Tailoring the ovarian stimulation protocol is based on assessment the daily gonadotrophins dose, that is built on several parameters including woman age, body mass index, the cause of infertility and assessment of ovarian reserve (basal FSH, antral follicle count and/or antimullerian hormone level).

Furthermore, the daily dose of gonadotrophins is adjusted based on the response of patient to gonadotrophins that reflected by monitoring serum estradiol level and the number and size of recruited follicles [11].

The aim of this study was to compare the efficacy of biosimilar FSH preparations and highly purified urinary FSH in ovulation induction in PCOs infertile women.

\section{Patients and Methods}

This a prospective observational comparative study that was conducted on 100 infertile women with PCOs according to Rotterdam 2003 criteria [12], (a) Oligo- and/or anovulation (b) Clinical and/or biochemical signs of hyperandrogenism (c) Polycystic ovaries (Two of three criteria needed). The patients were recruited from outpatient infertility clinic of Obstetrics and Gynecology department, Faculty of Medicine, South Valley University, Egypt from February 2017 to March 2018.

Verbal counseling and well-informed written consent were obtained from all cases agreed to participative in this study according to the committee of Medical Ethics of Faculty of Medicine, South Valley University. Exclusion criteria include (previous ovarian drilling, history of hyperstimulation syndrome, presence of other factors of infertility as tubal or male factors).

\section{Methods}

The patients in both groups were underwent simple ovarian induction, they were classified randomly into 2 groups as regarding to induction drugs used (Group I: included 50 women received biosimilar FSH agent (Gonapure 75iu, Magic pharma, administered intramuscularly) and Group II: included 50 women received highly purified urinary FSH (Fostimon 75iu, IPSA, administered intramuscularly). Biosimilar FSH preparation or highly purified urinary FSH were started in the second day of spontaneous or induced cycle. 75 iu (one ampoule) was used as starting dose in both groups and all cases were monitored for follicular development by transvaginal ultrasound (Medison, 7.5 $\mathrm{mH}$ ) the doses of gonadotrophins in both groups was increased or decreased according to the rate of follicular growth, follicular monitoring was each other day when leading follicles reach 14-16 $\mathrm{mm}$, when leading follicle achieved $18-20 \mathrm{~mm} 5000-10000 \mathrm{IU}$ of HCG was injected intramuscularly as a trigger for final follicular maturation. Endometrial thickness and its pattern were assessed for each patient at time of HCG. Each patient was advised to have intercourse 12 hours after HCG triggering and repeated 36 hours later.

\section{Outcome Measures}

primary outcome was measuring the number of mature follicles in the 2 ovaries, total doses and duration of gonadotrophins stimulation, endometrial thickness and its pattern on the day of HCG triggering. The secondary outcome assessed the pregnancy (chemical and clinical) rate, complications of ovarian stimulations in both groups, incidence of early pregnancy loss. Serum quantitative of $\beta$-hCG was assayed in cases had missed period for 3-5 days after expected menses. Hormonal support for luteal phase was done with $400 \mathrm{mg}$ prontogest administered vaginally daily at bed time and continued till pregnancy test time and positive cases were observed at outpatient antenatal care unit the end of first trimester, any maternal complications like hyper-stimulation syndrome was recorded and admitted to our hospital for management.

\section{Statistical Analysis}

Recorded data were analyzed using the statistical package for social sciences, version 20.0 (SPSS Inc., Chicago, Illinois, USA). Quantitative data were expressed as mean \pm standard deviation (SD). Qualitative data were expressed as frequency and percentage. Chi-square $\left(\chi^{2}\right)$ was used in order to compare proportions between two qualitative parameters and t-test was used when comparing between two means. P-value $<0.05$ was considered significant and if $\mathrm{p}$ value $<0.001$ was considered as highly significant but if $\mathrm{P}$-value $>0.05$ was considered insignificant.

\section{Results}

There was a mildly statistically significant difference between biosimilar FSH preparations group and highly purified urinary FSH in the mean \pm SD of gonadotrophins doses used, the duration of stimulation, the number of mature follicles with $\mathrm{p}<0.05$, but there was a highly statistically significant difference in endometrial thickness at time of HCG triggering with $\mathrm{p}<0.01$ (Table1-3). There was a mildly statistically significant difference between the 2 groups in pregnancy rates (chemical and clinical) with $\mathrm{p}<0.05$, but there were no statistically significant differences in the incidence of first trimester pregnancy loss between the 2 groups with $\mathrm{p}>0.05$ (table 3). As regarding to incidence of complications occurred in the 2 groups, there was mildly statistically significant differences in development of hyperstimulation syndrome (mild form) and twins pregnancies with $\mathrm{p}<0.05$ (Figure 1). 
Table 1: Patient characteristics.

\begin{tabular}{|c|c|c|c|}
\hline & Group I $(N=50)$ & Group II $(\mathrm{N}=44)$ & $P$ value \\
\hline Age in years (mean \pm SD) & $26.96 \pm 2.73$ & $26.67 \pm 3.85$ & NS \\
\hline BMI Kg/m² $($ mean $\pm S D)$ & $27.45 \pm 1.34$ & $27.31 \pm 1.15$ & NS \\
\hline $\mathrm{AMH} n \mathrm{n} / \mathrm{ml}($ mean $\pm \mathrm{SD})$ & $1.0 \pm 0.3$ & $9.7 \pm 0.2$ & NS \\
\hline Basal AFC (mean \pm SD) & $4.6 \pm 2.3$ & $4.3 \pm 2.1$ & NS \\
\hline Day 2 FSH $(\mathrm{mIU} / \mathrm{mL})($ mean \pm SD) & $9.3 \pm 3.4$ & $9.6 \pm 3.2$ & NS \\
\hline Day 2 E2 (pg/mL) (mean \pm SD) & $34.2 \pm 16.2$ & $35.6 \pm 18.4$ & NS \\
\hline Infertility Duration (yrs) (mean \pm SD) & $8.77 \pm 2.58$ & $8.33 \pm 3.41$ & NS \\
\hline Basal FSH (IU/ml) (mean \pm SD) & $5.46 \pm 1.35$ & $5.57 \pm 2.04$ & NS \\
\hline Basal LH (IU/ml) (mean \pm SD) & $10.57 \pm 3.67$ & $11 \pm 4.11$ & NS \\
\hline Testosterone (pg/dl) (mean \pm SD) & $1.1 \pm 0.41$ & $1.09 \pm 0.42$ & NS \\
\hline Basal AFC (mean \pm SD) & $1.08+0.64$ & $1.07+0.66$ & NS \\
\hline Ovarian volume (ml) $($ mean \pm SD) & $6.7+2.1$ & $6.9+2.2$ & NS \\
\hline Menstrual cycle pattern (No \%) & $17(34 \%)$ & $19(38 \%)$ & NS \\
\hline Regular Irregular & $33(66 \%)$ & $31(62 \%)$ & \\
\hline
\end{tabular}

NS= Non-significant $(p$ value $<0.05)$

Table 2: Outcome of ovarian stimulation in Group I and Group II.

\begin{tabular}{|c|c|c|c|}
\hline & Group I $(\mathrm{N}=50)$ & Group II $(\mathrm{N}=50)$ & P-value \\
\hline Duration of gonadotrophins stimulation (days) (mean \pm SD) & $10.7 \pm 1.4$ & $13.3 \pm 1.7$ & $S$ \\
\hline Number of gonadotrophins ampoules used (mean \pm SD) & $8.8 \pm 1.3$ & $11.9 \pm 1.6$ & S \\
\hline Number of follicles $>18 \mathrm{~mm}$ (day of HCG) & $2.9 \pm 1.6$ & $1.8 \pm 1.7$ & S \\
\hline Endometrial thickness at day of HCG injection in mm (mean \pm SD) & $9.6 \pm 1.7$ & $7.9 \pm 1.5$ & $\mathrm{~S}^{*}$ \\
\hline
\end{tabular}

$S=$ significant $(p$ value $>0.05){ }^{*}=$ highly significant $(p$ value $<0.01)$

Table 3: Pregnancy outcome in Group I and Group II.

\begin{tabular}{|c|c|c|c|}
\hline & Group I (N=50) & Group II (N=50) & P- value \\
\hline Chemical pregnancy rate per initiated cycle (No \%) & $11(22.0)$ & $8(16.0)$ & $\mathrm{S}$ \\
\hline Clinical pregnancy rate per initiated cycle (No \%) & $8(16.0)$ & $6(12.0)$ & $\mathrm{S}$ \\
\hline First trimester pregnancy loss (No \%) & $1(2)$ & $1(2)$ & NS \\
\hline
\end{tabular}

NS= Non-significant $(p$ value $<0.05), S=$ Significant $(p$ value $>0.05)$

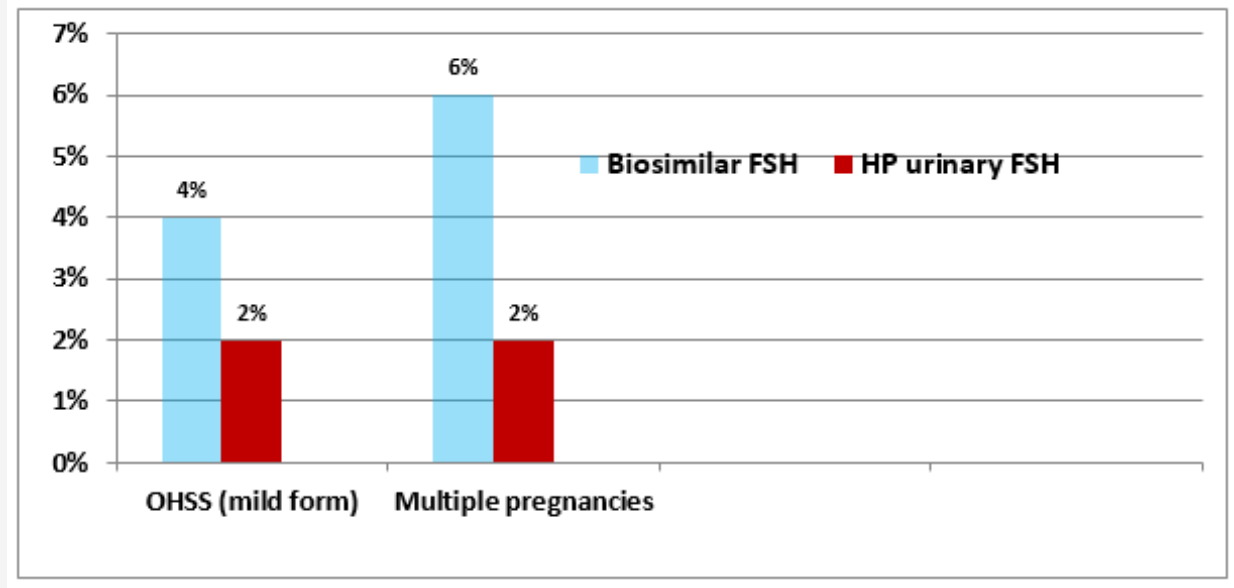

Figure 1:Comparison between Group I and II as regard to stimulation drugs complications.

\section{Discussion}

Polycystic ovary syndrome represents the most common etiology of anovulatory infertility. There are many methods for ovulation induction in cases of PCOS, which can eventually provide successful live births. Each method varies as regard to its effectiveness and its aggressiveness [13]. Both recombinant FSH and urinary FSH had been supposed to be more suitable for the controlled ovarian hyperstimulation protocols in PCOS infertile 
women because these patients had elevated levels of endogenous LH [14]. Biosimilar FSH preparations are not exact copies and could not be considered as generic versions of the reference product. They are considered as a regulatory synonym that could facilitate a fast track introduction of FSH preparation to the controlled ovarian stimulation armamentarium [15].

The biosimilar FSH preparations have non-clinical (pharmacological, pharmacokinetic, and toxicological) profiles, that looks like those of the originator FSH and it has been licensed for the all indications of reference product [16]. In this study we aimed to compare between the efficacies of biosimilar FSH preparations and highly purified urinary FSH in ovulation induction in infertile women with polycystic ovary syndrome, we found no significant differences between biosimilar FSH preparations group and highly purified FSH group as regard to women's age, BMI, basal hormones (FSH, LH and FSH/LH ratio), antimullerian hormone (AMH), basal antral follicle count (AFC), ovarian volume and menstrual pattern with $\mathrm{p}$ value $<0.05$.

As regarding to the mean \pm SD of doses (number of ampoules) and durations (in days) of ovarian stimulation were $(10.7 \pm 1.4$ $\& 8.8 \pm 1.3$ in group I and $13.3 \pm 1.7 \& 11.9 \pm 1.6$ in group II) which was statistically significant with $p$ value $<0.05$. The mean \pm SD of developed follicles $>18 \mathrm{~mm}$ was $(2.89 \pm 1.6$ in biosimilar FSH group and $1.83 \pm 1.77$ in HP-uFSH) that had a mildly significance with $p$ value $<0.05$, these results was less than what had been reported by Rettenbacher et al. [17] (10.8 \pm 5.11 in biosimilar FSH group). In our study the mean \pm SD of endometrial thickness at time of HCG triggering was $(9.6 \pm 1.7$ in biosimilar FSH group and 7.9 \pm 1.5 in HPuFSH group) that had a highly statistically significant difference ( $p$ value $<0.01$ ), In Sohrabvand et al. [14], the endometrial thickness in HP-uFSH group was $(10.36 \pm 1.35)$ which was higher than own. In our study biosimilar FSH preparations group had a higher pregnancy rates (chemical and clinical) than HP urinary FSH $(22 \%$ \& 16\% in biosimilar FSH group and $16 \%$ and $12 \%$ in HP-uFSH group) with $\mathrm{p}$ value $<0.01$ ), but there were no statistically significant differences in the incidence of first trimester pregnancy loss between the 2 groups with $\mathrm{p}$ value $>0.05$.

As regarding to incidence of the complications that developed in the 2 studied groups, we had found ovarian hyperstimulation syndrome (mild form) in our study was (4\% in biosimilar group versus $2 \%$ HP-FSH group) these results was nearly the same reported by Gizzo et al [18], where OHSS had recorded in 14 (5.6\%) of the biosimilar FSH group but no case developed severe or moderate degree of OHSS in both groups. Multiple gestations (twins' pregnancies) was recorded $6 \%$ in group I and $2 \%$ in group II that had a highly statistically significant with $\mathrm{p}$ value $<0.05$. The main goal in simple ovulation induction is to achieve minimal number of mature follicles and induced ovulation of follicles, preferable one to obtain a single pregnancy without OHSS.

A higher bioactivity of the biosimilar FSH preparations in ovulation induction in this study because they had a lower total number of ampoules used for stimulation beside had a shorter duration of stimulation, also the cost of drug should be in mind in its preferring over HP-uFSH. Many previous studies compared recombinant FSH with urinary $\mathrm{FSH}$, in this study we tried to compare biosimilar FSH preparations with urinary FSH, we had found biosimilar FSH efficacy and safety were better than urinary FSH in ovulation induction for PCOs patients beside its low cost in comparison to referral recombinant FSH drugs.

\section{Conclusion}

Biosimilar FSH preparations were better than the highly purified urinary FSH in ovulation induction in PCOs infertile women (short duration and low doses of stimulation, significant increase in growing follicles number, enhancement in endometrial thickness and increased pregnancy rate). So, this study recommends the use of biosimilar preparations of FSH in ovulation induction in PCOs infertile women.

\section{Acknowledgement}

None.

\section{Conflict of Interest}

No Conflict of Interest.

\section{References}

1. Goldenberg N, Glueck C (2008) Medical therapy in women with polycystic ovarian syndrome before and during pregnancy and lactation. Minerva Ginecol 60(1): 63-75.

2. Bachmann A, Rabe T, Bühler K, Griesinger G, Sanger N (2015) The Gonadotropins FSH and LH and their use in Adult Women - an Overview. J Reproduktionsmed Endokrinol 12 (4): 361-375.

3. Herrler A, Beneke H (2008) Considerations and recommendations for patient safety in the application of modern gonadotropin preparations. J Reproduktionsmed Endokrinol 5: 132-137.

4. Biffoni M, Battaglia A, Borrelli F, Cantelmo A, Galli G, et al. (1994) Allergenic potential of gonadotrophic preparations in experimental animals: relevance of purity. Hum Reprod 9: 1845-1848.

5. Albano C, Smitz J, Camus M, Riethmuller Winzen H, Siebert Weigel M, et al. (1996) Hormonal pro®le during the follicular phase in cycles stimulated with a combination of human menopausal gonadotrophin and gonadotrophin-releasing hormone antagonist (Cetrorelix). Hum Reprod 11: 2114-2118.

6. van Weissenbruch MM, Schoemaker HC, Drexhage HA, Schoemaker J (1993) Pharmaco-dynamics of human menopausal gonadotrophin (HMG) and follicle-stimulating hormone (FSH). The importance of the FSH concentration in initiating follicular growth in polycystic ovary-like disease. Hum Reprod 8(6): 813-821.

7. Hayden CJ, Balen AH, Rutherford AJ (1999) Recombinant gonadotrophins. British J Obstet Gynaecol 106: 188-196.

8. US Food and Drug Administration. Guidance for industry. Scientific considerations in demonstrating bio similarity to a reference product.

9. Mellstedt H, Niederwieser D, Ludwig $H$ (2008) The challenge of biosimilars. Ann Oncol 19(3): 411-419.

10. Bridges SL Jr, White DW, Worthing AB, Gravallese EM, O’Dell JR (2018) The Science Behind Biosimilars. Entering a New Era of Biologic Therapy. Arthritis Rheumatol 70(3): 334-344.

11. Seifer DB, Tal R (2015) Personalized prediction of live birth: are we there yet? Fertil Steril 104(2): 283-285.

12. Rotterdam ESHRE/ASRM-Sponsored PCOS Consensus Workshop Group (2004) Revised 2003 consensus on diagnostic criteria and long-term health risks related to polycystic ovary syndrome. Fertil Steril 81(1): 19-25.

13. Perales-Puchalt A, Legro RS (2013) Ovulation induction in women with polycystic ovary syndrome. Steroids 78(8): 767-772. 
14. Sohrabvand F, Sheikhhassani S, Bagheri M, Haghollahi F, Shabihkhani M, et al. (2012) Comparison of highly purified urinary versus recombinant FSH: Effect on ART outcomes in polycystic ovary syndrome. Iran J Reprod Med 10(3): 229-236.

15. Orvieto R, Seifer DB (2016) Biosimilar FSH preparations- are they identical twins or just siblings? Reprod Biol Endocrinol : 14:32.

16. Sanghvi S (2015) Assessment of bemfola: biosimilar follitropin alfa. J Form Med Man :4-6.
17. Rettenbacher M, Andersen AN, Garcia-Velasco JA, Sator M, Barri P, et al. (2015) A multi-centre phase 3 study comparing efficacy and safety of Bemfola versus Gonal-f in women undergoing ovarian stimulation for IVF. Reprod Biomed Online: 30(5): 504-513.

18. Gizzo S, Garcia-Velasco JA, Heiman F, Ripellino C, Bühler K (2016) A cost-effectiveness evaluation comparing originator follitropin alfa to the biosimilar for the treatment of infertility. Int J Women's Health 8:683689. 